\title{
A high speed $X-Y$ nanopositioner with integrated optical motion sensing
}

Cite as: Rev. Sci. Instrum. 90, 035002 (2019); https://doi.org/10.1063/1.5055715

Submitted: 10 September 2018 . Accepted: 09 February 2019 . Published Online: 18 March 2019

Priyanka Gupta, P. Piyush, R. Sriramshankar, and G. R. Jayanth
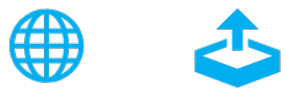

\section{ARTICLES YOU MAY BE INTERESTED IN}

Improved gating device of time-of-flight ion mass analyzer for ion sources

Review of Scientific Instruments 90, 033305 (2019); https://doi.org/10.1063/1.5088576

Capacitive sensor for fast pulsed voltage monitor in transmission line

Review of Scientific Instruments 90, 035107 (2019); https://doi.org/10.1063/1.5050276

Measurement of the injecting time of picosecond laser in indirect-drive integrated fast ignition experiments using an x-ray streak camera

Review of Scientific Instruments 90, 033504 (2019); https://doi.org/10.1063/1.5050039

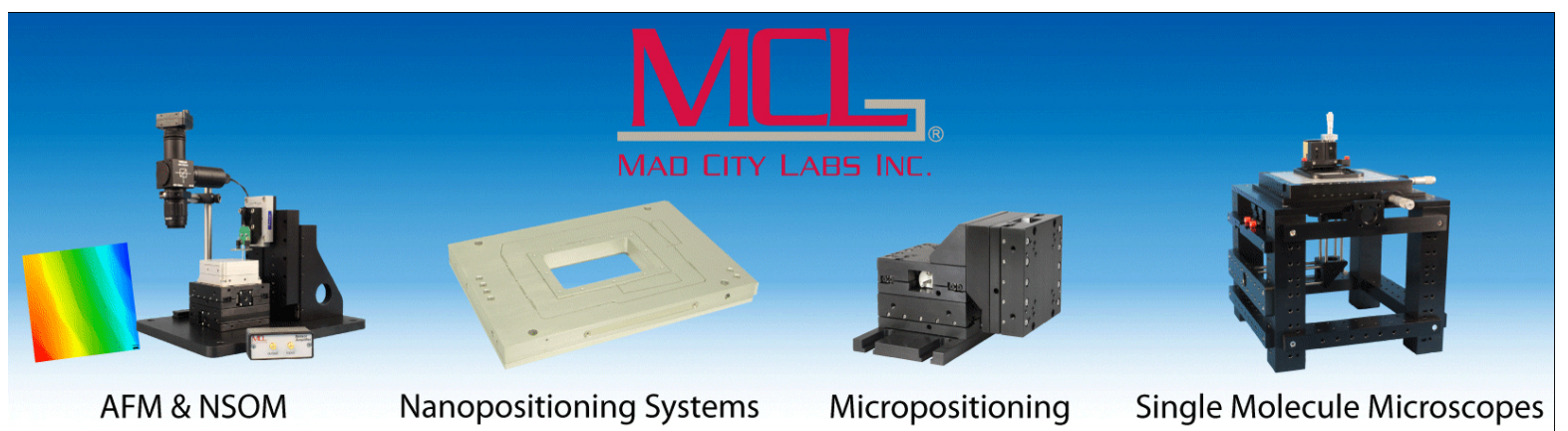




\title{
A high speed $X-Y$ nanopositioner with integrated optical motion sensing
}

\author{
Cite as: Rev. Sci. Instrum. 90, 035002 (2019); doi: 10.1063/1.5055715 \\ Submitted: 10 September 2018 - Accepted: 9 February 2019 • \\ Published Online: 18 March 2019
}

Priyanka Gupta, ${ }^{\text {a) }}$ P. Piyush, ${ }^{\text {a) }}$ R. Sriramshankar, and G. R. Jayanth

AFFILIATIONS

Department of Instrumentation and Applied Physics, Indian Institute of Science, Bangalore 560012, India

a) Contributions: Priyanka Gupta and P. Piyush contributed equally to this work.

\begin{abstract}
High speed in-plane (X-Y) nanopositioners are of central importance in scanning probe microscopy for performing fast imaging and manipulation. Reducing the size of the nanopositioning stage improves the response speed of the positioner but also introduces challenges in integration of conventional motion sensors. This paper presents the design and development of a novel high speed flexure-guided, piezoelectrically actuated nanopositioner with integrated optical beam deflection-based motion sensing. The sensing strategy eliminates spatial constraints even for small stages. A simple lumped-parameter model is proposed for the nanopositioner. Subsequently, the model is used to design and fabricate the nanopositioner. The measurement system is integrated with the nanopositioning stage and is employed to characterize the quasi-static and dynamic response of the stage. Finally, the in-plane motion measurements are employed to control the stage when it is commanded to track both slow- and fast-varying position signals. In both cases, the use of control is shown to significantly improve the tracking accuracy.
\end{abstract}

Published under license by AIP Publishing. https://doi.org/10.1063/1.5055715

\section{INTRODUCTION}

$\mathrm{X}-\mathrm{Y}$ nanopositioners are employed to provide in-plane motion to a sample with nanometer scale resolution in applications such as nanometrology, ${ }^{1}$ nanofabrication, ${ }^{2}$ and material property characterization. $^{3}$ With growing demands on throughput and spatial accuracy in these applications, ${ }^{4,5}$ nanopositioners are required to deliver ever faster and more precise motion. This necessitates them to possess mechanical bandwidths of several kilohert $z^{6}$ and nanometer scale resolution over motion range of several micrometers, while ensuring minimal uncertainties and errors. ${ }^{7}$ To provide such fast and precise motion, piezoelectric actuators are commonly used owing to their high resolution, motion bandwidth, and stiffness. $^{8}$

Since common in-plane nanopositioners, such as the piezotube $^{9}$ and the shear in-plane piezo-actuators, ${ }^{10}$ suffer from issues of either limited bandwidth or limited range, alternate designs based on flexure-guided stages have been explored. In flexure-guided nanopositioning stages, uniaxial stack piezo-actuators of adequate motion range and bandwidth are employed for actuation along both $\mathrm{X}$ and Y-axis, with negligible cross coupling, ${ }^{11}$ and their motion is either guided or amplified by flexures. The design of flexure-guided stages is classified into two broad configurations based on the arrangement of the $\mathrm{X}$ - and $\mathrm{Y}$-actuators: serial-kinematic mechanism, where the stage and one in-plane actuator is driven by another orthogonally located actuator, ${ }^{12}$ and parallel-kinematic mechanism, where two orthogonally located actuators move the stage directly, independent of each other. ${ }^{13}$ Among the two configurations, the serial kinematic mechanism offers less cross-coupling, but its bandwidth along one axis is much smaller than the bandwidth along the other. One such stage has been reported to achieve an eigenfrequency of $24.2 \mathrm{kHz}$ along the fast scanning direction with a travel range of $9 \mu \mathrm{m} .{ }^{12}$ On the other hand, parallel kinematic mechanisms achieve high bandwidths along both X- and Y-directions, and so the scan direction can be chosen arbitrarily. Such stages are suitable for implementation of several scanning strategies other than the conventional raster-pattern that are of interest in high speed scanning. ${ }^{14}$ Such a stage ${ }^{15}$ with a resonant frequency of $2.7 \mathrm{kHz}$ and a travel range of $25 \mu \mathrm{m}$ has been reported that employed an amplification lever for piezo-actuator displacement. Another design ${ }^{16}$ reports to have achieved a resonant frequency of $22 \mathrm{kHz}$ and a travel range of $13 \mu \mathrm{m}$ using a push-pull configuration for the piezo-actuators along each axis. Further improvements in speed demand further reduction in the inertia of the stage. 
Regardless of the specific stage design, piezo-actuated stages suffer from inherent non-linearities, such as hysteresis and creep, ${ }^{17}$ and therefore necessitate real-time position feedback and control. The most noteworthy techniques for achieving fast and highresolution motion measurement are contact-type sensing elements based on capacitive transducers ${ }^{18,19}$ and non-contact optical measurement. ${ }^{20,21}$ However, to achieve high measurement resolution from the capacitive transducer, the area of its electrodes need to be relatively large, typically about $1 \mathrm{~cm}^{2}$. In combination with the fact that the electrode needs to be fixed to a rigid support, this implies that as the stage dimensions shrink, the electrodes themselves dominate the mass of the nanopositioning stage and ultimately limit the positioning bandwidth. ${ }^{22}$ The alternate technique of using inter-digital capacitors is not generally adopted owing to challenges in fabrication. ${ }^{23}$ Therefore, non-contact optical measurement techniques, such as interferometry and optical beam deflection (OBD), are preferred. While interferometry ensures high resolution and bandwidth, the complexity of its optics makes it impractical for small positioning stages, especially for measurement of two-axis in-plane motion. ${ }^{24}$ Optical beam deflection, which is another high resolution measurement technique, is conventionally employed only for measurement of out-of-plane rotation. Previously we reported integration of a curved mirror on to the moving stage to achieve high sensitivity to in-plane translation motion. ${ }^{25}$ Since the area of the curved mirror is decided only by the motion range and the spot diameter, the mirror itself can be made small enough to be integrated even to MEMS stages. ${ }^{25}$ Thus, unlike a conventional capacitive sensor, the curved mirror contributes to negligible inertia, especially when integrated with macro-scale stages.

In this paper, we first propose a design for a flexure-guided nanopositioning stage that ensures a high eigen-frequency and an adequate actuation range. In particular, the dimensions of the nanopositioning stage are chosen to be less than a centimetre, in order to minimize its inertia. Since the compact size precludes the use of capacitive sensing, an optical beam deflection based in-plane motion measurement system is integrated with the nanopositioning stage. The optical motion measurement system is employed to characterize the actuation range and eigen-frequency of the nanopositioner. Furthermore, in combination with iterative learning control, the overall system has been shown to enable tracking of fast scan waveforms with about $70 \%$ lesser hysteresis compared to the open-loop system.

Section II gives the design and modeling of the in-plane nanopositioning stage. Section III discusses the fabrication of the nanopositioner, its experimental evaluation, and its control by means of the in-plane optical motion measurement system. Section IV presents the conclusions.

\section{DESIGN AND MODELING OF THE X-Y NANOPOSITIONING SYSTEM}

Figure 1(a) shows the schematic of the proposed high speed $\mathrm{X}-\mathrm{Y}$ nanopositioning system. It comprises a nanopositioning stage actuated by two stack piezo-actuators aligned along the $\mathrm{X}$ - and $\mathrm{Y}$ axis, respectively, and coupled to the stage by means of flexures. The flexures employ a parallel kinematic design to ensure high speed along both the axes of motion. The resulting in-plane motion of the stage is measured by means of optical beam deflection. To perform

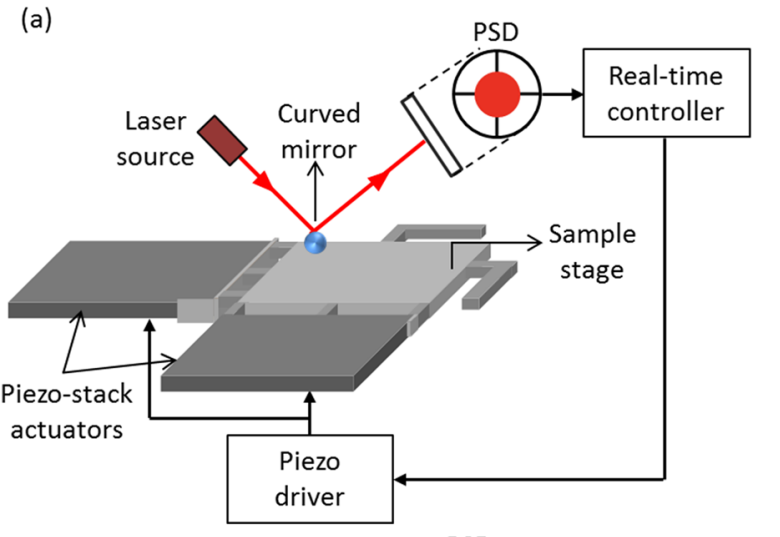

(b)
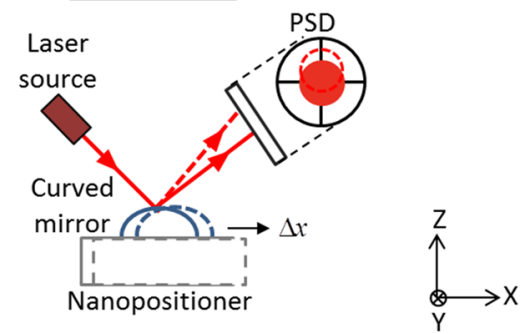

FIG. 1. (a) Schematic of the developed X-Y nanopositioning system. (b) Principle of integrated optical motion sensing.

the measurement, a laser beam is focused on a curved mirror that is rigidly integrated with the nanopositioning stage, and the reflected light is captured by a position sensitive detector (PSD). Thus, when the stage undergoes in-plane displacement, the local slope of the surface at the site of laser incidence changes, thereby resulting in deflection of the reflected beam [Fig. 1(b)]. Under optimal conditions, the measurement system has been shown previously to enable achieving a shot-noise limited resolution of a few picometers in a bandwidth of $1 \mathrm{kHz} \cdot{ }^{25}$ Owing to the small size of the mirror, its contribution to the overall inertia of the stage would be negligible, even when the stage itself is small.

Figure 2(a) shows the mechanical design of the overall X-Y nanopositioner. It comprises the nanopositioning stage $S$ whose motion is guided by three pairs of flexures $F_{1}, F_{2}$, and $F_{3}$, with one member of each pair used to guide motion along a particular axis.

The stage is chosen to be hollow in order to reduce its mass. Owing to the symmetry in the arrangement of the flexure of each type, their roles are described only along one axis: The flexure $F_{1}$ comprises a pair of slender beams laterally attached on either side of the cap upon which the moving end of the piezo-actuator would rest [Fig. 2(b)]. The role of this flexure is to constrain the lateral motion of the piezo-actuator and to guide its motion along the longitudinal axis of the actuator. The flexure $F_{2}$ comprises 3 slender guided beams that longitudinally couple the piezo-actuators to the nanopositioning stage [Fig. 2(c)]. The flexures coupling each piezo-actuator are designed to achieve high stiffness along the direction of motion of the piezo-actuator and low stiffness along the lateral direction. The flexure $F_{3}$ comprises a slender beam, $F_{3 b}$ attached orthogonally to a cantilever structure, $F_{3 c}$ [Fig. $2(\mathrm{~d})$ ]. Together, they serve to 
(a)
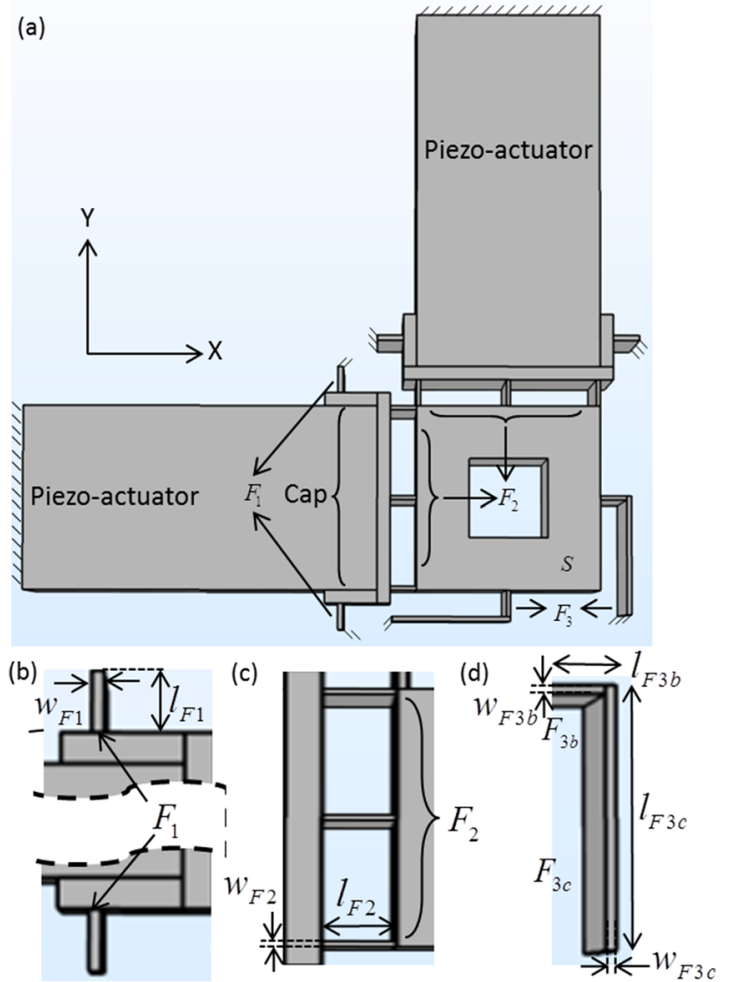

FIG. 2. The mechanical design of (a) the overall $X-Y$ nanopositioner, (b) flexure $F_{1}$, (c) flexure $F_{2}$, and (d) flexure $F_{3}$.

constrain the out-of-plane (Z-) motion of the stage, without significantly affecting its in-plane motion. Accordingly, the thicknesses of the beam and the cantilever are both significantly higher than their widths. The cantilever is designed to ensure that the flexure possesses low stiffness along the longitudinal axis of the piezo-actuator, while the beam serves to achieve low stiffness in the lateral direction.

In order to estimate the motion range and the approximate eigen-frequency, a lumped-parameter model is proposed for the nanopositioner. The piezo-actuator is modeled to have a mass $M_{P}$ and a stiffness $k_{P}$. The flexures are assumed to contribute only to the compliance, while the stage is assumed to contribute only to inertia. Designating $M_{S}$ as the mass of the stage, $k_{\text {lat }}^{F j}$ as the lateral stiffness of the flexure $F_{j}(j=1,2,3)$, and $k_{l o n}^{F j}$ as its longitudinal stiffness, the lumped-parameter model of the nanopositioner along one axis is shown in Fig. 3.

In Fig. 3, $x_{i p}$ refers to the longitudinal displacement of the piezo-actuator for an applied voltage of $V_{i p}$, when it is constrained

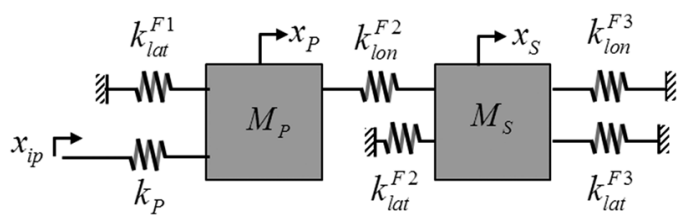

FIG. 3. Schematic of the lumped-parameter model of the nanopositioner.
TABLE I. Expressions for the various parameters of the lumped-parameter model for the nanopositioner.

\begin{tabular}{lc}
\hline \hline Parameter & Expression \\
\hline$k_{P}$ & $E_{P} A_{c P} / \ell_{P}$ \\
$k_{\text {lat }}^{F 1}$ & $2 E t_{F 1} w_{F 1}^{3} / \ell_{F 1}^{3}$ \\
$k_{\text {lon }}^{F 2}$ & $3 E t_{F 2} w_{F 2} / \ell_{F 2}$ \\
$k_{\text {lat }}^{F 2}$ & $3 E t_{F 2} w_{F 2}^{3} / \ell_{F 2}^{3}$ \\
$k_{\text {lat }}^{F 3}$ & $E t_{F 3 b} w_{F 3 b}^{3} / \ell_{F 3 b}^{3}$ \\
$k_{\text {lon }}^{F 3}$ & $E t_{F 3 c} w_{F 3 c}^{3} / 4 \ell_{F 3 c}^{3}$ \\
$M_{p}$ & $\rho_{P} \ell_{P} A_{c P} / 3$ \\
\hline \hline
\end{tabular}

only at its base. $x_{P}$ refers to the displacement when the piezoactuator is connected to the flexure-guided stage. $x_{i p}$ is obtained from the actuation gain $G_{P Z T}$ of the piezo-actuator as $x_{i p}=G_{P Z T} V_{i p}$. The expressions for the different parameters in the lumpedparameter model can be obtained from the dimensions of the piezoactuator, the beams of flexures $F_{1}$ and $F_{2}$, and the beam and cantilever elements of flexure $F_{3}$ and are summarized in Table I. It is worth noting that in obtaining the expressions for $k_{\text {lat }}^{F 3}$ and $k_{\text {lon }}^{F 3}$, the flexure $F_{3 b}$ is modeled approximately as a single guided beam, while the flexure $F_{3 c}$ is modeled approximately as a cantilever beam.

In Table I, $\rho_{P}$ and $E_{P}$ represent the density and Young's modulus of the piezo-actuator, respectively, while $E$ represents Young's modulus of the flexures. Similarly, $\ell_{P}$ and $A_{c} P$ represent the length and cross-sectional area of the piezo-actuator, respectively, and $\ell_{F j}$, $w_{F j}$, and $t_{F j}$ represent the length, width, and thickness of the flexure elements $F_{j}(j=1,2,3 b, 3 c)$.

The model can be employed to obtain the dynamic equation governing the motion of the stage and is given by

$$
\left[\begin{array}{cc}
M_{P} & 0 \\
0 & M_{S}
\end{array}\right]\left[\begin{array}{c}
\ddot{x}_{P} \\
\ddot{x}_{S}
\end{array}\right]+\left[\begin{array}{ll}
k_{11} & k_{12} \\
k_{21} & k_{22}
\end{array}\right]\left[\begin{array}{c}
x_{P} \\
x_{S}
\end{array}\right]=\left[\begin{array}{c}
k_{P} x_{i p} \\
0
\end{array}\right],
$$

where

$$
k_{11}=k_{\text {lat }}^{F 1}+k_{\text {lon }}^{F 2}+k_{P}, k_{12}=-k_{\text {lon }}^{F 2}, k_{21}=-k_{\text {lon }}^{F 2}
$$

and

$$
k_{22}=k_{\text {lat }}^{F 2}+k_{\text {lon }}^{F 3}+k_{\text {lat }}^{F 3}+k_{\text {lon }}^{F 2} .
$$

The dynamic model in Eq. (1) can be employed to obtain the relationship between the quasi-static displacement $x_{S}$ of the stage and the input $x_{i p}$ as

$$
x_{S}=\left[\begin{array}{ll}
0 & 1
\end{array}\right]\left[\begin{array}{ll}
k_{11} & k_{12} \\
k_{21} & k_{22}
\end{array}\right]^{-1}\left[\begin{array}{c}
k_{P} \\
0
\end{array}\right] x_{i p} .
$$

The lowest eigen-frequency of the dynamic model would also be the estimate of the lowest eigen-frequency of the stage.

\section{FABRICATION, CHARACTERIZATION, AND CONTROL OF THE X-Y NANOPOSITIONING SYSTEM}

This section discusses the fabrication, characterization, and motion control of the developed flexure-guided nanopositioning 
stage. Section III A describes the fabrication of the nanopositioner, while Sec. III B describes simultaneous two-axis motion measurement and dynamic characterization of the stage. Section III C reports motion control of the developed stage under both quasi-static actuation and actuation at higher frequencies.

\section{A. Design and fabrication of the nanopositioner}

In the developed nanopositioner, the nanopositioning stage was chosen to be $7 \mathrm{~mm} \times 7 \mathrm{~mm}$ in area and the desired motion range was chosen to be about $8 \mu \mathrm{m}$. The small dimensions of the stage were chosen to ensure an adequately low mass for the stage. The stage and the flexures were chosen to be made of aluminum. The dynamic lumped-parameter model described in Sec. II was employed to obtain the dimensions of the flexures. Table II summarizes these dimensions.

A commercially available stack piezo-actuator (P 887.31, Physik Instrumente $\mathrm{GmbH}$ ) was employed to actuate the nanopositioner. Prior to its use, the actuation gain of the piezo-actuator, when it is constrained only at its base, was characterized by using a laser Doppler vibrometer and was found to be $63 \mathrm{~nm} / \mathrm{V}$.

Figure 4(a) shows the Computer Aided Design (CAD) model of the nanopositioner. The nanopositioning stage was fabricated using wire electrical discharge machining [Fig. 4(b)]. In the fabricated stage, the flexures were attached to a thick aluminum frame, which, in turn, was screwed down firmly to the optical bread board. Figures 4(c) and 4(d) show the photographs of the flexures $F_{2}$ and $F_{3}$, respectively

TABLE II. Dimensions of the various flexures.

\begin{tabular}{lccc}
\hline \hline & $\ell_{F j}(\mathrm{~mm})$ & $w_{F j}(\mathrm{~mm})$ & $t_{F j}(\mathrm{~mm})$ \\
\hline$F_{1}, F_{2}, F_{3 b}$ & 1 & 0.2 & 7 \\
$F_{3 c}$ & 5.25 & 0.4 & 7 \\
\hline \hline
\end{tabular}

(a)

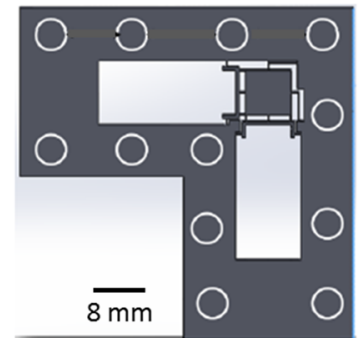

(c)

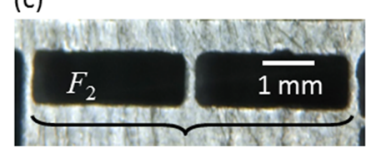

(b)

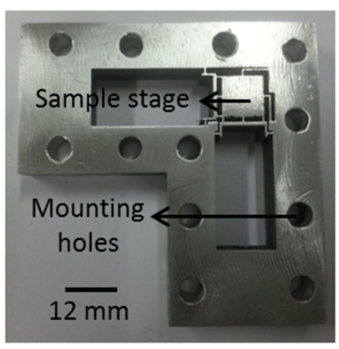

(d)

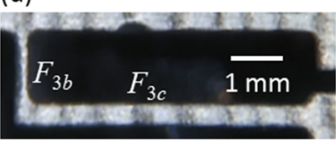

FIG. 4. (a) The CAD model of the in-plane nanopositioner. (b) Photograph of the fabricated flexure-guided nanopositioning stage. (c) Micro-photograph of the flexure $F_{2}$. (d) Micro-photograph of the flexure $F_{3}$.
Upon fabrication of the flexure-guided stage, the piezo-actuator was mounted within the stage by employing the arrangement shown in Fig. 5(a), wherein one end of the piezo-actuator was pressed against the cap to which the flexure $F_{1}$ was connected. The other end was supported by a rectangular block whose area of cross section was slightly larger than that of the piezo-actuator. The rectangular block was pushed against the piezo-actuator by means of a screw, and the block ensured that the pressure applied by the screw on the actuator is uniformly distributed. To ensure application of point-load on the block, a V-groove was fabricated on the back surface of the block and a steel ball was positioned within the groove, so that the screw made direct contact only with the steel ball. To ensure that the screw does not slip, especially during high-frequency actuation, it was passed through a threaded nylon plug. Figure 5(b) shows a photograph of the nanopositioner with the piezo-actuators mounted in it. Figures 5(c) and 5(d) show the photographs of the V-groove and the cap, respectively.

(a)

(b)
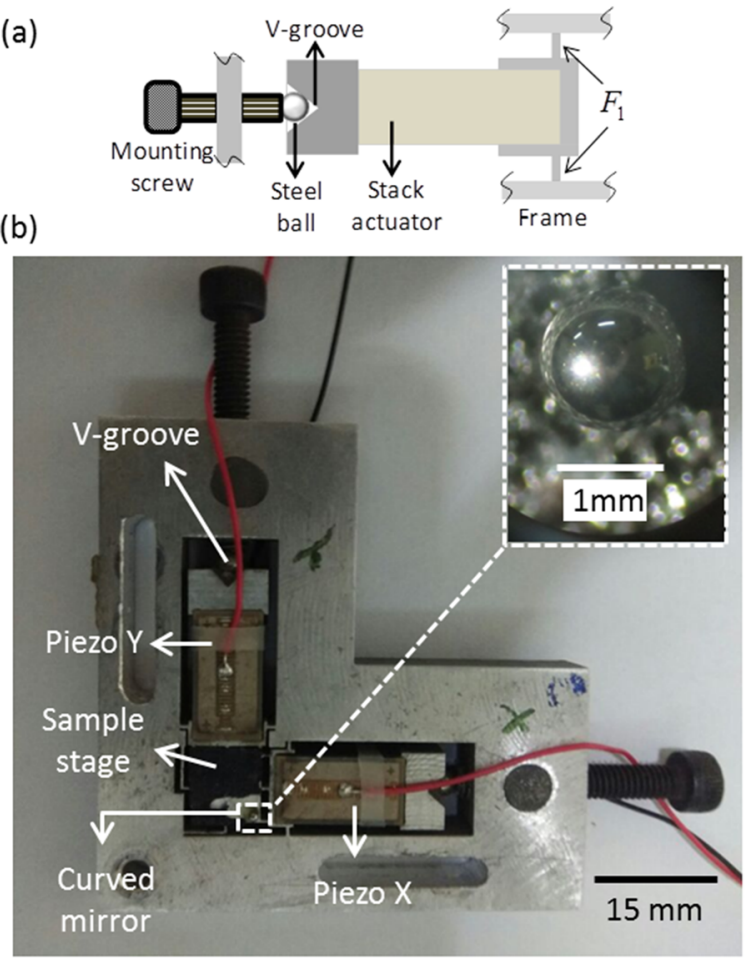

(c)

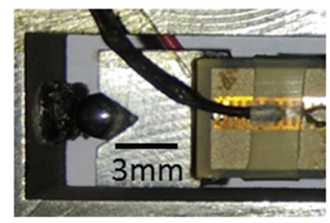

(d)

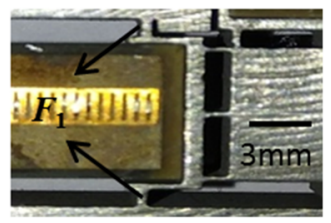

FIG. 5. (a) Schematic showing the arrangement for mounting the stack piezoactuators. (b) Photograph of the developed positioner with the piezo-actuator mounted in it along both axes. (c) Photograph of the V-groove. (d) Photograph of the cap and the flexure $F_{1}$. 


\section{B. Motion measurement and dynamic characterization of the nanopositioner}

In order to measure the motion of the stage by employing optical beam deflection, a reflective stainless steel sphere [Material: SS 1.4034(AISI 420c), Grade 10, Nanoball GmbH] of diameter $1 \mathrm{~mm}$ was rigidly glued to the surface of the nanopositioning stage. Figure 6(a) shows a schematic of the experimental setup that was employed for measuring the displacement of the in-plane nanopositioning stage.

A laser beam was launched through a stereo microscope (SMZT745, Nikon) on the sphere. The reflected light was captured by a position sensitive detector (S5991-01, Hamamatsu). The output of the detector was conditioned and acquired by a real-time controller (DS-1103, dSPACE GmbH). A photograph of the developed setup is shown in Fig. 6(b).

In order to calibrate the in-plane sensitivity and measurement range of the developed optical measurement system, the nanopositioning stage was mounted on a commercial nanopositioner (NanoCube P-611.3S, Physik Instrumente $\mathrm{GmbH}$ ) and the output of the measurement system was recorded to known two-axis in-plane displacements provided using the NanoCube. The response of the PSD captured along the X- and Y- axis is plotted in Figs. 7(a) and 7 (b), respectively. The responses along both the axes are linear for the respective input commands and the calibrated sensitivities along both the axes are almost identical to each other, i.e., $0.3 \mathrm{~V} / \mu \mathrm{m}$ along the $\mathrm{X}$-axis and $0.35 \mathrm{~V} / \mu \mathrm{m}$ along the $\mathrm{Y}$-axis. The cross-axis sensitivities of the system, namely, $0.025 \mathrm{~V} / \mu \mathrm{m}$ along the $\mathrm{Y}$-axis and $0.038 \mathrm{~V} / \mu \mathrm{m}$ along the $\mathrm{X}$-axis, are approximately 8 and 14 times smaller than the sensitivities along the $\mathrm{X}$-axis and $\mathrm{Y}$-axis, respectively. In order to determine the measurement range of the system, the output of the measurement system was recorded in response to a large input displacement from the commercial nanopositioner. Figures 7(c) and 7(d) plot the responses of the system for $\pm 14 \mu \mathrm{m}$ input displacements along the two in-plane axes. The responses are however not linear, and the sensitivity is seen to reduce at larger displacements. The linear measurement range was determined as the displacement up to which the measurement sensitivity is at least $90 \%$ of the sensitivity for small displacements. Based on this criterion, the linear measurement range of the system is found to be $\pm 8 \mu \mathrm{m}$ along the two axes. The nonlinearity in measurement at larger displacements results primarily from the large displacement of the (a)

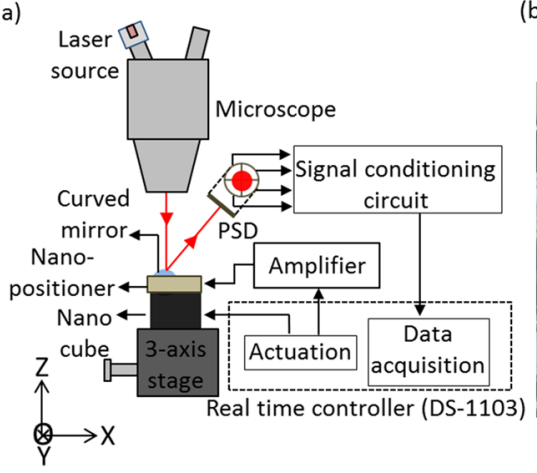

(b)

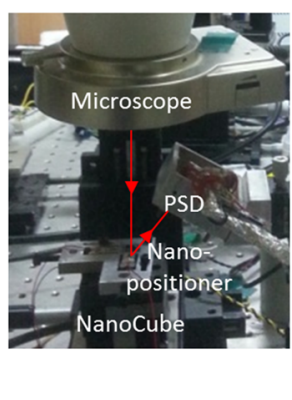

FIG. 6. (a) Schematic of the in-plane motion measurement system based on optical beam deflection. (b) Photograph of the developed setup. (a)

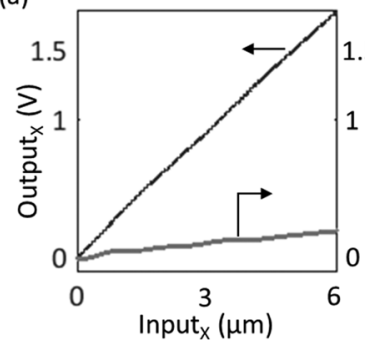

(c)

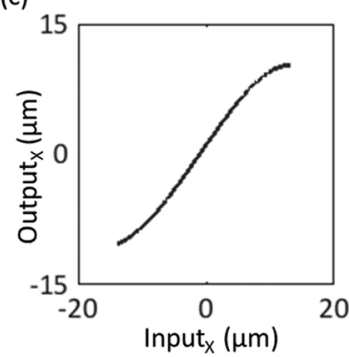

(b)

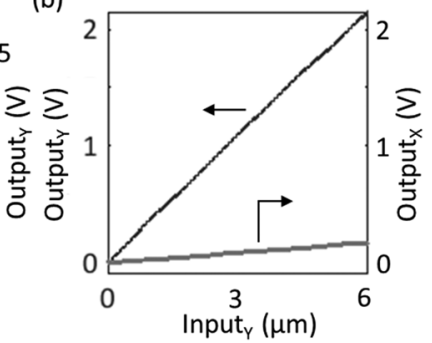

(d)

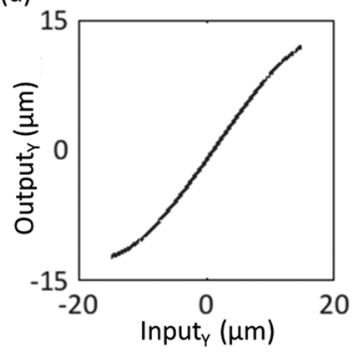

FIG. 7. Plots showing (a) calibrated sensitivity along the $X$-axis, (b) calibrated sensitivity along the $Y$-axis, (c) measurement range of the system along the $X$-axis, and (d) measurement range of the system along the $\mathrm{Y}$-axis.

laser spot on the detector. The theoretical dependence of the output voltage from the PSD on the spot displacement over it is reported in Ref. 27.

Subsequent to calibration of the measurement system, the stage was fixed firmly to the optical bread-board, and the quasi-static actuation gain and frequency response of the stage were characterized. In order to obtain the actuation gain, the nanopositioning stage was actuated by a $0.25 \mathrm{~Hz}, 30 \mathrm{~V}$ peak triangular voltage signal and the displacement of the stage was measured. The resulting actuation gain of the stage was found to be $48 \mathrm{~nm} / \mathrm{V}$ along the $\mathrm{X}$-axis and $45 \mathrm{~nm} / \mathrm{V}$ along the $\mathrm{Y}$-axis. Since the actuation gain of the unconstrained piezo-actuator was $63 \mathrm{~nm} / \mathrm{V}$, the reduction in actuation gain is about $24 \%$. For the chosen dimensions of the piezo-actuator and the flexures, the lumped-parameter model in Eq. (2) predicts the reduction to be $12 \%$. The difference between the two can be attributed to tolerances in the fabrication of the flexure-guided stage. Based on the estimated actuation gain and the permissible voltage limit for the piezo-actuator of $100 \mathrm{~V}$, an actuation range of $4.8 \mu \mathrm{m}$ along the $\mathrm{X}$-axis and $4.5 \mu \mathrm{m}$ along the $\mathrm{Y}$-axis was obtained.

After characterizing the quasi-static actuation gain of the stage, its frequency response was obtained along the $\mathrm{X}$ - and $\mathrm{Y}$-axis. In order to perform the experiment, the stage was actuated first along the $\mathrm{X}$-axis and subsequently along the $\mathrm{Y}$-axis with a sinusoidal input whose frequency was swept from $100 \mathrm{~Hz}$ to $10 \mathrm{kHz}$. Two lockin amplifiers were implemented in the real-time controller using MATLAB $^{\circledR}$ SIMULINK ${ }^{\mathrm{TM}}$ to measure the response amplitudes from the two output channels of the PSD. The sampling frequency of the real-time controller was set to be $60 \mathrm{kHz}$. By multiplying the acquired amplitudes with the inverse of the calibrated sensitivity matrix, the motion of the stage along the actuated axis and the cross-axis was determined. The characterized dynamics of the stage is presented as the magnitude responses of four transfer functions $T_{i j}(i, j \equiv X, Y)$, 
(a)

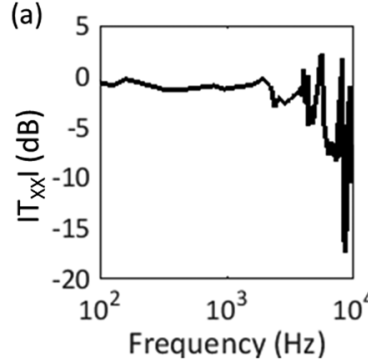

(c)

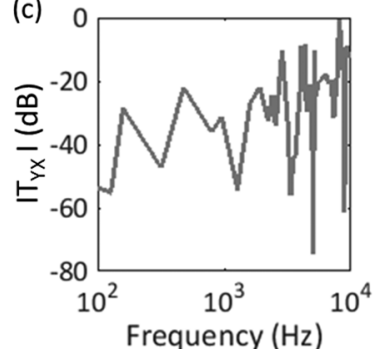

(b)

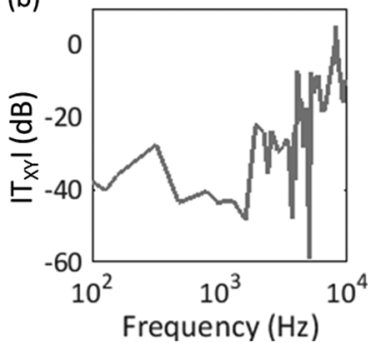

(d)

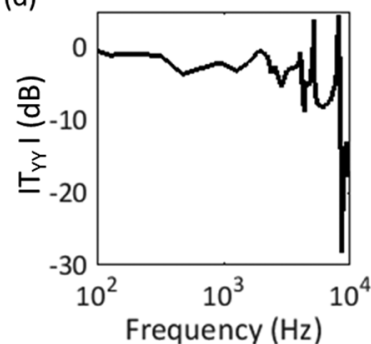

FIG. 8. Plots showing the normalized magnitude responses of the transfer functions relating (a) the output displacement measured along the $X$-axis to the input displacement applied along the X-axis, (b) the output displacement measured along the $\mathrm{X}$-axis to the input displacement applied along the Y-axis, (c) the output displacement measured along the $Y$-axis to the input displacement applied along the $\mathrm{X}$-axis, and (d) the output displacement measured along the $Y$-axis to the input displacement applied along the $Y$-axis.

which relate the motion along axis $i(i \equiv X, Y)$ to the input applied along axis $j(j \equiv X, Y)$ (Fig. 8). The magnitudes of the transfer functions $T_{i X}$ and $T_{i Y}(i \equiv X, Y)$ have been normalized with respect to the quasi-static actuation gains along the $\mathrm{X}$-axis and $\mathrm{Y}$-axis, respectively. Figure 8 shows that the dynamic responses of the stage along both the actuation axes are similar with resonant peaks at $4 \mathrm{kHz}, 5.2 \mathrm{kHz}$, and $8 \mathrm{kHz}$. The cross-axis motion of the developed nanopositioning stage is also seen to be small. Moreover, the characterized bandwidth of the stage is adequate to employ it in high speed scanning probe microscopy without significantly exciting its resonant dynamics.

\section{Motion control of the in-plane positioning stage}

The measured in-plane displacement of the nanopositioning stage was employed to control the stage for both high and low frequency reference signals. To control the stage for applications requiring low frequency reference signals, a feedback control system was designed by employing an integral controller of gain $K_{I}=$ 80 [Fig. 9(a)]. A slow triangular reference waveform was provided to actuate the stage, and its response was recorded both in openloop and in closed-loop. Figure 9(b) plots the input-output relationship for the two cases and shows that in open-loop, the response exhibits significant hysteresis of over $15.68 \%$. The positioning accuracy, which is decided by the half-width of the hysteretic loop, is therefore about $92 \%$. In contrast, the closed-loop response reveals reduction in the hysteresis to $3.25 \%$, i.e., by a factor of five.

In order to demonstrate simultaneous two-axis in-plane motion measurement capability of the system, the stage was commanded to track a circular reference trajectory of diameter $1 \mu \mathrm{m}$.
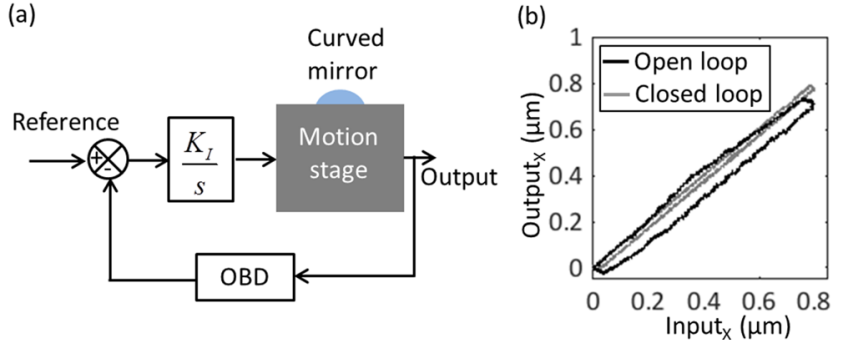

(c)

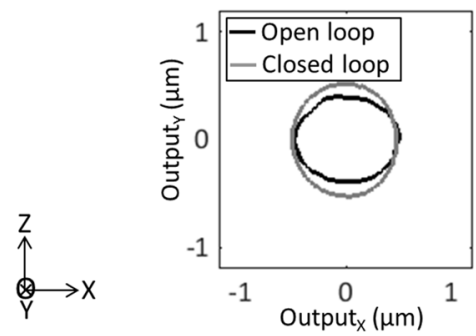

FIG. 9. (a) Block diagram representation of the closed-loop control of the nanopositioning stage under quasi-static actuation. (b) Input-output relationship with and without the implementation of closed-loop control. (c) Motion of the developed nanopositioning stage, in open-loop and in closed-loop, while tracking a circular trajectory.

To perform this experiment, the sensitivities of the system were first obtained and employed to estimate, and subsequently control, the displacements of the nanopositioner along the two axes. The commanded reference had a frequency of $0.1 \mathrm{~Hz}$. First, the stage was commanded in open-loop and the displacements were recorded. Subsequently, its motion was controlled by employing feedback control using integral controllers of gain 80 along each axis. The measured displacements under open- and closed-loop actuation of the developed nanopositioner are shown in Fig. 9(c). It can be observed from the plot that under open-loop actuation, the stage could not track the reference well, whereas with the feedback control, it was able to track the reference with significantly higher accuracy. This experiment reveals that the developed stage along with the integrated measurement system can be utilized for precise positioning of samples in the X-Y plane.

Subsequently, in order to demonstrate high positioning bandwidth of the stage, it was actuated with a triangular voltage signal of $500 \mathrm{~Hz}$ frequency and its motion was controlled by employing an iterative learning control (ILC) technique. ${ }^{26}$ Such a high scanning frequency enables samples to be imaged at 5 frames/s with an image resolution of $100 \times 100$ pixels. A schematic showing the implementation of this technique is presented in Fig. 10(a). In this technique, the error $e_{m}$ between the measured waveform and the commanded waveform during the $m$ th period of a periodic waveform is corrected in the $m+1$ th period by applying the correction $u_{m+1}=u_{m}+K e_{m}$, where $K$ is the actuation gain. Figure 10(b) plots the input-output relationships at the start of the implementation and after three iterations. It can be observed that in the first iteration of attempting to track a sinusoidal actuation signal at $500 \mathrm{~Hz}$, the positioning hysteresis was $17.3 \%$. With 3 iterations after implementing the control strategy, the hysteresis was reduced to $5.2 \%$. Thus, within just about $6 \mathrm{~ms}$ 
(a)

(b)

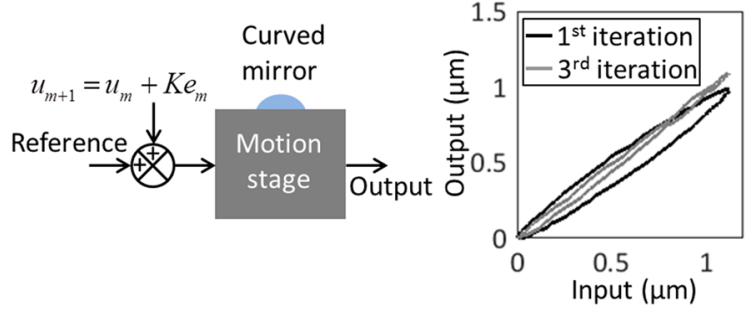

FIG. 10. (a) Block diagram representation of the implementation of an iterative learning control technique. (b) Input-output relationship with and without the implementation of the iterative control strategy.

duration, substantial improvement could be achieved in tracking the commanded waveform.

\section{CONCLUSION}

This paper presented the design and development of a high speed flexure-guided in-plane nanopositioner with integrated motion sensing. The measurement system is especially beneficial for smaller stage sizes, where measurement of motion using other techniques with high resolution is challenging. The proposed design for the nanopositioner is simple and enables high positioning bandwidth about both axes. A lumped-parameter dynamic model was proposed for the nanopositioner in order to facilitate its design. Subsequently, the nanopositioner was designed, fabricated, and integrated with the optical motion measurement system. The experimentally characterized quasi-static range was found to agree well with the prediction of the lumped-parameter model. The measurement system was also employed to characterize the dynamics of the nanopositioner. Finally, the measurements were used in combination with conventional feedback control and with iterative learning control, to improve the tracking performance of the nanopositioner to both slow and fast reference signals.

\section{ACKNOWLEDGMENTS}

This material is based on the work supported by the ISRO-IISc Space Technology Cell under Grant No. ISTC0369 and IMPRINT India Initiative under Grant No. IMPR0006. Any opinions, finding, conclusions, or recommendations expressed in this material are those of the authors and do not necessarily reflect the views of the
ISRO-IISc Space Technology Cell or IMPRINT India Initiative. The experiments were carried out at Applied Photonics Initiative, Indian Institute of Science.

\section{REFERENCES}

${ }^{1}$ F. J. Giessibl, Rev. Mod. Phys. 75(3), 949 (2003).

${ }^{2}$ A. A. Tseng, A. Notargiacomo, and T. P. Chen, J. Vac. Sci. Technol., B: Microelectron. Nanometer Struct. 23(3), 877 (2005).

${ }^{3}$ H. J. Butt, B. Cappella, and M. Kappl, Surf. Sci. Rep. 59, 1 (2005).

${ }^{4}$ T. Ando, Nanotechnology 23, 062001 (2012).

${ }^{\mathbf{5}}$ E. T. Herruzo, A. P. Perrino, and R. Garcia, Nat. Commun. 5, 3126 (2014).

${ }^{6}$ K. K. Leang and A. J. Fleming, Asian J. Control 11(2), 144 (2009).

${ }^{7}$ D. Croft, G. Shed, and S. Devasia, J. Dyn. Syst., Meas., Control 123, 35 (2001).

${ }^{8}$ G. Y. Gu, L. M. Zhu, C. Y. Su, H. Ding, and S. Fatikow, IEEE Trans. Autom. Sci. Eng. 13(1), 313 (2016).

${ }^{9}$ S. O. R. Moheimani, Rev. Sci. Instrum. 79, 071101 (2008).

${ }^{10}$ See https://www.physikinstrumente.com for PICA Shear Actuators, P-111P-153.

${ }^{11}$ Y. K. Yong, S. O. R. Moheimani, B. J. Kenton, and K. K. Leang, Rev. Sci. Instrum. 83, 121101 (2012).

${ }^{12}$ B. J. Kenton and K. K. Leang, IEEE/ASME Trans. Mechatronics 17(2), 356 (2012).

${ }^{13}$ A. J. Fleming and K. K. Leang, Design, Modeling and Control of NanoPositioning Systems, Advances in Industrial Control (Springer International Publishing, Switzerland, 2014), pp. 57-100.

${ }^{14}$ Y. K. Yong, S. O. R. Moheimani, and I. R. Petersen, Nanotechnology 21, 365503 (2010).

${ }^{15}$ Y. K. Yong, S. S. Aphale, and S. O. R. Moheimani, IEEE Trans. Nanotechnol. 8(1), 46 (2009).

${ }^{16}$ G. Schitter, K. J. Åström, B. E. DeMartini, P. J. Thurner, K. L. Turner, and P. K. Hansma, IEEE Trans. Control Syst. Technol. 15(5), 906 (2007).

${ }^{17}$ S. Devasia, E. Eleftheriou, and S. O. R. Moheimani, IEEE Trans. Control Syst. Technol. 15(5), 802 (2007).

${ }^{18}$ S. Polit and J. Dong, IEEE/ASME Trans. Mechatronics 16(4), 724 (2011).

${ }^{19}$ Y. K. Yong, A. J. Fleming, and S. O. R. Moheimani, IEEE/ASME Trans. Mechatronics 18(3), 1113 (2013).

${ }^{20}$ J.-P. Monchalin, J.-D. Aussel, R. Heon, C. K. Jen, A. Boudreault, and R. Bernier, J. Nondestr. Eval. 8(2), 121 (1989).

${ }^{21}$ B. Pan, K. Qian, H. Xie, and A. Asundi, Meas. Sci. Technol. 20(6), 062001 (2009).

${ }^{22}$ A. J. Fleming, Sens. Actuators, A 190, 106 (2013).

${ }^{23}$ J. I. Lee, X. Huang, and P. B. Chu, J. Microelectromech. Syst. 18(3), 660-670 (2009).

${ }^{24}$ K. Zhu, B. Guo, Y. Lu, S. Zhang, and Y. Tan, Optica 4(7), 729 (2017).

${ }^{25}$ R. Sriramshankar, R. S. M. Mrinalini, and G. R. Jayanth, Rev. Sci. Instrum. 84, 105001 (2013).

${ }^{26}$ D. A. Bristow, M. Tharayil, and A. G. Alleyne, IEEE Control Syst. 26(3), 96-114 (2006).

${ }^{27}$ T. Fukuma and P. S. Jarvis, Rev. Sci. Instrum. 77, 043701 (2006). 